\title{
The sign problem across the QCD phase transition
}

\author{
Jens O. Andersen ${ }^{a, b}$, Lars T. Kyllingstad ${ }^{b}$, and Kim Splittorff ${ }^{c}$ \\ ${ }^{a}$ Niels Bohr International Academy, Blegdamsvej 17, DK-2100 Copenhagen, Denmark \\ ${ }^{b}$ Department of Physics, Norwegian University of Science and Technology, Høgskoleringen 5, N-7491 Trondheim, Norway \\ ${ }^{c}$ Niels Bohr Institute, Blegdamsvej 17, DK-2100 Copenhagen, Denmark
}

(Dated: November 19, 2018)

\begin{abstract}
The average phase factor of the QCD fermion determinant signals the strength of the QCD sign problem. We compute the average phase factor as a function of temperature and baryon chemical potential using a two-flavor NJL model. This allows us to study the strength of the sign problem at and above the chiral transition. It is discussed how the $U_{A}(1)$ anomaly affects the sign problem. Finally, we study the interplay between the sign problem and the endpoint of the chiral transition.

PACS numbers: 12.39.-x, 11.30.-j, 12.38.Gc, 12.38.Lg
\end{abstract}

\section{INTRODUCTION}

Suppose that there was no sign problem in QCD at nonzero baryon chemical potential. Then, by means of lattice $\mathrm{QCD}$, it would be possible directly to study the phase diagram of QCD. In particular, it would be of great interest to get a direct computation of the preferred ground state of strongly interacting matter for low temperature and chemical potential of order $\Lambda_{\mathrm{QCD}}$. This region is relevant for dense stars, and a number of theoretically motivated ground states has been proposed [1, 2, 3, 4] — see Ref. [5] for a review. Clearly, a reliable lattice QCD test of these theoretical predictions could lead to substantial progress. It would also be of relevance to study chemical potentials and temperatures in the range of $100-400 \mathrm{MeV}$ where ultrarelativistic heavyion collisions are conducted.

In order to show that it is the sign problem which is the main obstacle for such first principle numerical investigations of the QCD phase diagram, let us for a moment consider phase-quenched QCD. In the phasequenched version, one replaces the fermion determinant by its absolute value. Standard lattice techniques can therefore be applied. Studies of this theory on the lattice show a rich phase diagram [6, 7, 8 ]. For two flavors the phase-quenched theory is realized by coupling the chemical potential to the third component of the isospin rather than to the quark number [9], and the phases obtained numerically can be understood physically. For example, the phase quenched lattice data show that a second-order phase transition takes place at zero temperature once the isospin chemical potential reaches half of the pion mass [6, 7, [8]. This is understood to be the transition into a phase with a Bose condensate of pions. Besides offering direct insights into an alternative direction of the QCD phase diagram, the phase quenched studies also demonstrate that without the sign problem it would be possible also to study nonzero quark chemical potential by means of lattice QCD.

While a direct solution of the QCD sign problem eludes us at present, progress has been made to circumvent the sign problem 10, 11, 12, 13, 14, 15, 16, 17, 18, 19, 20,
21, 22, 23, 24, 25, 26]. The success of these approaches is linked to the degree of cancellations which take place due to the sign problem. One way to measure the degree of such cancellations is by means of the average of the phase factor

$$
e^{2 i \theta}=\frac{\operatorname{det}\left(D+\mu \gamma_{0}+m\right)}{\operatorname{det}\left(D+\mu \gamma_{0}+m\right)^{*}} .
$$

If the average of the phase factor is tiny then severe cancellations take place in the path integral defining the QCD partition function and the validity of lattice computations should be examined carefully. Studies of the average phase factor are therefore important in order to understand lattice gauge computations which circumvent the sign problem.

Chiral perturbation theory has been used to predict the behavior of the average phase factor 27, 28, 29]. These predictions have been confirmed by lattice QCD measurements. Of course, as the temperature approaches the critical one, chiral perturbation theory is no longer valid since the more massive modes are no longer Boltzmann suppressed. For example, it has been demonstrated in Ref. 31] that the effect of the nucleons on the average phase factor can be tracked in lattice QCD simulations just below the critical temperature.

The purpose of the present study is to examine the average phase factor also at and above the chiral phase transition, where we can not trust chiral perturbation theory. In order to carry out such a study we necessarily need to rely on a specific model. Here we have chosen the two-flavor NJL model. This is perhaps the most widely used framework to model aspects of the QCD phase diagram [32, 33, 34, 35, 36, 37, 38]. For a review, see Ref. [39].

Since the phase factor is what separates phase quenched QCD from full QCD, the expectation value of the phase factor in the phase quenched theory is simply

$$
\left\langle e^{2 i \theta}\right\rangle=\frac{Z_{1+1}}{Z_{1+1^{*}}},
$$

where the subscript $1+1$ refers to the ordinary two-flavor 
theory and $1+1^{*}$ refers to the two-flavor phase quenched theory (since it has a flavor and a conjugate flavor).

In the present paper we will evaluate this ratio within the NJL model at mean field level. The general structure of the saddle point approximation gives

$$
\left\langle e^{2 i \theta}\right\rangle=\frac{Z_{1+1}}{Z_{1+1^{*}}}=\frac{\sqrt{\operatorname{det} H_{1+1^{*}}}}{\sqrt{\operatorname{det} H_{1+1}}} e^{-\beta V\left(\Omega_{1+1}-\Omega_{1+1^{*}}\right)},
$$

where $\Omega$ is the mean field free energy density and $H$ is the Hessian matrix describing the fluctuations at the saddle point.

For small values of the chemical potential, the mean free energies in the $1+1$ theory and the $1+1^{*}$ theory are identical. In this case, the prefactor - the ratio of the Hessian determinants - determines the average phase factor. For $\mu>m_{\pi} / 2$, the phase quenched theory enters the Bose condensed phase mentioned above. The two meanfield free energies are therefore different in this phase.

This effect was first understood within chiral perturbation theory. It is also present when the average phase factor is evaluated within a chiral random matrix model [40] and also results from an exact computation in the $\epsilon$-regime of chiral perturbation theory [41, 42].

The $U_{A}(1)$ axial anomaly affects the order of the chiral phase transition at zero chemical potential [43]. A recent study [44] shows that the location of the endpoint of the first order chiral transition can be highly sensitive to the strength of the $U_{A}(1)$ breaking. Here we compute the effects of the $U_{A}(1)$ breaking on the average phase factor within the two-flavor NJL model.

Since the strength of the sign problem sets a practical upper limit for lattice QCD it is crucial to understand the value of the average phase factor at the endpoint. Here we discuss the interplay between the endpoint and the average phase factor. Perhaps somewhat surprisingly we show that the average phase factor grows (indicating a milder sign problem) as one approaches the endpoint. The reason is that the massless mode associated with the endpoint induces a pole in the prefactor of Eq. (3).

The paper is organized as follows. In Sec. II, we briefly discuss the two-flavor NJL model and its symmetries. In Sec. III, we study the average phase factor without axial symmetry breaking, and in Sec. IV, we investigate the effects of axial symmetry breaking. In Sec. V, we look at the interplay between the sign problem and the endpoint of the chiral transition, and in Sec. VI we summarize our results.

\section{THE NJL MODEL AND THE AVERAGE PHASE FACTOR}

In this section, we briefly discuss the two-flavor NJL model and our conventions. Note in particular that we are including the prefactor in our mean-field calculation of the partition function, cf. Eq. (28).

The fermionic part of the two-flavor QCD Lagrangian at finite baryon chemical potential and finite isospin chemical potential is

$$
\bar{\psi}\left(D-m+\mu_{B} B \gamma_{0}+\mu_{I} I_{3} \gamma_{0}\right) \psi,
$$

where $B=\operatorname{diag}(1 / 3,1 / 3), I_{3}=\tau_{3} / 2$, and $D$ is the QCD fermion operator at zero chemical potentials. Since the Lagrangian is quadratic in the fermionic fields, the fermions can be integrated out exactly, yielding the fermion determinant

$$
\begin{aligned}
& \operatorname{det}\left(D+m-\mu_{B} B \gamma_{0}-\mu_{I} I_{3} \gamma_{0}\right) \\
& =\operatorname{det}\left(D+m-\mu \gamma_{0}-\delta \mu \gamma_{0}\right) \\
& \quad \times \operatorname{det}\left(D+m-\mu \gamma_{0}+\delta \mu \gamma_{0}\right),
\end{aligned}
$$

where the chemical potentials $\mu$ and $\delta \mu$ are

$$
\mu \equiv \frac{\mu_{B}}{3} \quad \text { and } \quad \delta \mu \equiv \frac{\mu_{I}}{2} .
$$

$\mu$ is thus the quark chemical potential.

As mentioned in the introduction, phase-quenched QCD with two flavors is realized by setting $\mu=0$ and interpreting $\delta \mu$ as the quark chemical potential instead.

The NJL Lagrangian is given by [39]

$$
\mathcal{L}=\mathcal{L}_{0}+(1-\alpha) \mathcal{L}_{1}+\alpha \mathcal{L}_{2},
$$

where the various terms are

$$
\begin{aligned}
& \mathcal{L}_{0}=\bar{\psi}\left(i \gamma^{\mu} \partial_{\mu}-m\right) \psi \\
& \mathcal{L}_{1}=G\left[(\bar{\psi} \psi)^{2}+(\bar{\psi} \boldsymbol{\tau} \psi)^{2}+\left(\bar{\psi} i \gamma^{5} \psi\right)^{2}+\left(\bar{\psi} i \gamma^{5} \boldsymbol{\tau} \psi\right)^{2}\right] \\
& \mathcal{L}_{2}=G\left[(\bar{\psi} \psi)^{2}-(\bar{\psi} \boldsymbol{\tau} \psi)^{2}-\left(\bar{\psi} i \gamma^{5} \psi\right)^{2}+\left(\bar{\psi} i \gamma^{5} \boldsymbol{\tau} \psi\right)^{2}\right] .
\end{aligned}
$$

In the two-flavor case, $\psi$ is an isospin doublet, i.e.

$$
\psi=\left(\begin{array}{l}
u \\
d
\end{array}\right)
$$

and $m=\operatorname{diag}\left(m_{u}, m_{d}\right)$ is the quark mass matrix. In the following, we take $m_{u}=m_{d}=m_{0}$. Also, $\boldsymbol{\tau}=\left(\tau_{1}, \tau_{2}, \tau_{3}\right)$, where the $\tau_{i} \mathrm{~s}$ are the Pauli matrices acting in isospin space.

The Lagrangian (77) is invariant under global $S U\left(N_{c}\right)$ and $U(1)_{B}$ symmetries. The latter corresponds to conservation of baryon number. The interaction terms $\mathcal{L}_{1}$ and $\mathcal{L}_{2}$ both have a a global $S U\left(N_{f}\right)_{\mathrm{L}} \times S U\left(N_{f}\right)_{\mathrm{R}}$, which is broken explicitly to a $S U\left(N_{f}\right)_{\mathrm{L}+\mathrm{R}}$ by a nonzero value of $m_{0}$. Finally, $\mathcal{L}_{1}$ has a $U(1)_{A}$ symmetry which is not shared by $\mathcal{L}_{2}$. This means that we can use the parameter $\alpha$ to control the strength of the axial symmetry violation in the model, and when $\alpha=0$, the Lagrangian (7) is $U(1)_{A}$ symmetric.

We introduce chemical potentials for baryon number density and isospin density by, respectively, adding the terms

$$
\begin{aligned}
\mathcal{L}_{B} & =\mu_{B} \bar{\psi} \gamma^{0} B \psi, \\
\mathcal{L}_{I} & =\mu_{I} \bar{\psi} \gamma^{0} I_{3} \psi .
\end{aligned}
$$


Using the definitions in Eq. (6), we can now write the Lagrangian as

$$
\begin{aligned}
\mathcal{L}= & \bar{\psi}\left(i \gamma^{\mu} \partial_{\mu}-m_{0}+\mu \gamma^{0}+\delta \mu \gamma^{0} \tau_{3}\right) \psi \\
& +G\left[(\bar{\psi} \psi)^{2}+\left(\bar{\psi} i \gamma^{5} \boldsymbol{\tau} \psi\right)^{2}\right] \\
& +(1-2 \alpha) G\left[(\bar{\psi} \boldsymbol{\tau} \psi)^{2}+\left(\bar{\psi} i \gamma^{5} \psi\right)^{2}\right]
\end{aligned}
$$

When $\alpha=1 / 2$, the terms in the scalar-isovector and pseudoscalar-isovector channels vanish, and one recovers the standard NJL Lagrangian.

Next, we introduce four auxiliary scalar fields $\sigma$ and $a_{i}$, and four pseudscalar fields $\eta$ and $\pi_{i},(i=1,2,3)$, by adding the following terms to the Lagrangian:

$$
\begin{aligned}
\mathcal{L}_{\text {aux }}= & -\frac{1}{4 G}\left[(\sigma-2 G \bar{\psi} \psi)^{2}+\left(\pi_{i}-2 G \bar{\psi} i \gamma^{5} \tau_{i} \psi\right)^{2}\right] \\
& -\frac{1-2 \alpha}{4 G}\left[\left(\eta-2 G \bar{\psi} i \gamma^{5} \psi\right)^{2}+\left(a_{i}-2 G \bar{\psi} \tau_{i} \psi\right)^{2}\right] .
\end{aligned}
$$

If we use the equation of motion for the auxiliary fields to eliminate them, we recover the original Lagrangian. The quartic interaction terms in the Lagrangian vanish and we obtain

$$
\begin{aligned}
\mathcal{L}=\bar{\psi}\left[i \gamma^{\mu} \partial_{\mu}-m_{0}+\mu \gamma^{0}+\delta \mu \gamma^{0} \tau_{3}-\sigma\right. \\
\left.-i \gamma^{5} \tau_{i} \pi_{i}-(1-2 \alpha)\left(i \gamma^{5} \eta+\tau_{i} a_{i}\right)\right] \psi \\
-\frac{1}{4 G}\left(\sigma^{2}+\pi_{i} \pi_{i}\right)-\frac{1-2 \alpha}{4 G}\left(\eta^{2}+a_{i} a_{i}\right)
\end{aligned}
$$

Based on the last term in Eq. (16), it is evident that we must require $\alpha \leq 1 / 2$ for the theory to be stable, which means that the $\mathrm{U}_{A}(1)$ symmetry is maximally violated for $\alpha=1 / 2$.

To allow for a chiral condensate $\langle\bar{\psi} \psi\rangle$ and a charged pion condensate $\left\langle\bar{\psi} i \gamma^{5} \tau_{1} \psi\right\rangle$, we introduce nonzero expectation values for the fields $\sigma$ and $\pi_{1}$. Expanding around these values, we have that

$$
\begin{aligned}
\sigma & \rightarrow \sigma-2 G\langle\bar{\psi} \psi\rangle, \\
\pi_{1} & \rightarrow \pi_{1}-2 G\left\langle\bar{\psi} i \gamma^{5} \tau_{1} \psi\right\rangle .
\end{aligned}
$$

For notational simplicity, we introduce the quantities

$$
\begin{aligned}
M & \equiv m_{0}-2 G\langle\bar{\psi} \psi\rangle \\
\rho & \equiv-2 G\left\langle\bar{\psi} i \gamma^{5} \tau_{1} \psi\right\rangle .
\end{aligned}
$$

We can now write the Lagrangian as

$$
\begin{aligned}
\mathcal{L}=\bar{\psi}\left[i \gamma^{\mu} \partial_{\mu}-M-i \gamma^{5} \tau_{1} \rho+\mu \gamma^{0}+\delta \mu \gamma^{0} \tau_{3}\right. \\
\left.-\sigma-i \gamma^{5} \tau_{i} \pi_{i}-(1-2 \alpha)\left(i \gamma^{5} \eta+\tau_{i} a_{i}\right)\right] \psi \\
-\frac{1}{4 G}\left(\sigma^{2}+\pi_{i} \pi_{i}\right)-\frac{1-2 \alpha}{4 G}\left(\eta^{2}+a_{i} a_{i}\right) .
\end{aligned}
$$

The pion condensate $\rho$ breaks parity and isospin symmetry.

Note that when $\alpha \neq 1 / 2$ we should, in general, allow for nonequal $\langle\bar{u} u\rangle$ and $\langle\bar{d} d\rangle$ condensates, since $\left\langle a_{3}\right\rangle=$
$-2 G(\langle\bar{u} u\rangle-\langle\bar{d} d\rangle)$. However, in this paper we will only consider the special cases $(\mu \neq 0, \delta \mu=0)$ and $(\mu=$ $0, \delta \mu \neq 0)$, and in both these cases $\langle\bar{u} u\rangle=\langle\bar{d} d\rangle$.

After applying the shifts in Eq. (??), the Lagrangian is quadratic in the fermion fields, which means that they can be explicitly integrated out. Thus, the partition function of the NJL model is

$$
Z_{\mathrm{NJL}}=\int \mathcal{D} \sigma \prod_{i=1}^{3} \mathcal{D} \pi_{i} \mathcal{D} \eta \prod_{j=1}^{3} \mathcal{D} a_{j} e^{-S_{\mathrm{eff}}\left[\sigma, \pi_{i}, \eta, a_{j}\right]},
$$

where the effective action is given by

$$
\begin{aligned}
& S_{\mathrm{eff}}\left[\sigma, \pi_{i}, \eta, a_{i}\right]=-\log \operatorname{det} K \\
& \quad+\frac{1}{4 G} \int d^{3} x \int_{0}^{\beta} d \tau\left[\sigma^{2}+\pi_{i} \pi_{i}+(1-2 \alpha)\left(\eta^{2}+a_{i} a_{i}\right)\right],
\end{aligned}
$$

and the matrix $K$ is defined as

$$
\begin{aligned}
K \equiv & \gamma_{\mu} P_{\mu}+m_{0}-\mu \gamma_{0}-\delta \mu \gamma_{0} \tau_{3}+\sigma \\
& +i \gamma_{5} \tau_{i} \pi_{i}+(1-2 \alpha)\left(i \gamma^{5} \eta+\tau_{i} a_{i}\right)
\end{aligned}
$$

The matrix $K$ has components in Dirac, color, and isospin space, as well as spacetime, and the determinant must be taken over all these spaces. In general this is highly nontrivial to do analytically, but it can be simplified somewhat by defining a matrix $K^{\prime}$ by

$$
K^{\prime} \equiv K \gamma_{0}
$$

Since $\operatorname{det}\left(\gamma_{0}\right)=1$, this matrix has the property that $\log \left(\operatorname{det} K^{\prime}\right)=\log (\operatorname{det} K)$. Unlike $K$, however, $K^{\prime}$ has all $p_{0}$ s on the diagonal, so it can be written as

$$
K^{\prime}=p_{0}+K^{\prime}\left(p_{0}=0\right) .
$$

Thus, the eigenvalues of $K^{\prime}$ (and $K$ ) can be written as

$$
\lambda_{i}=p_{0}+\varepsilon_{i}
$$

where $\varepsilon_{i}$ are the eigenvalues of the simpler matrix $K^{\prime}\left(p_{0}=0\right)$. The fermion part of the effective action is then

$$
\log \operatorname{det} K=\sum_{i} \log \left(p_{0}+\varepsilon_{i}\right)
$$

Since the original matrix $K$ is the inverse fermion propagator, we immediately identify $\varepsilon_{i}$ as the energies of the quasiparticles in the spectrum.

The values of the condensates $M$ and $\rho$ are found by minimizing the effective potential in the $(M, \rho)$ plane, i.e. by simultaneously solving the equations

$$
\frac{\partial S_{\mathrm{eff}}}{\partial M}=0, \quad \frac{\partial S_{\mathrm{eff}}}{\partial \rho}=0,
$$

while ignoring the fluctuations in the fields. 
Using the saddle-point approximation to evaluate the partition function at this minimum, we find that

$$
Z_{\mathrm{NJL}}=\frac{1}{\sqrt{\operatorname{det} H}} e^{-\beta V \Omega},
$$

where the thermodynamic potential $\Omega$ is the effective action evaluated at the saddle point and divided by the four-volume $\beta V . H$ is the Hessian matrix of $S_{\text {eff }}$, defined as

$$
H=\left(\begin{array}{cccc}
\frac{\partial^{2} S_{\text {eff }}}{\partial \sigma^{2}} & \frac{\partial^{2} S_{\text {eff }}}{\partial \sigma \partial \pi_{1}} & \frac{\partial^{2} S_{\text {eff }}}{\partial \sigma \partial \pi_{2}} & \frac{\partial^{2} S_{\text {eff }}}{\partial \sigma \partial \pi_{3}} \\
\frac{\partial^{2} S_{\text {eff }}}{\partial \pi_{1} \partial \sigma} & \frac{\partial^{2} S_{\text {eff }}}{\partial \pi_{1}^{2}} & \frac{\partial^{2} S_{\text {eff }}}{\partial \pi_{1} \partial \pi_{2}} & \frac{\partial^{2} S_{\text {eff }}}{\partial \pi_{1} \partial \pi_{3}} \\
\frac{\partial^{2} S_{\text {eff }}}{\partial \pi_{2} \partial \sigma} & \frac{\partial^{2} S_{\text {eff }}}{\partial \pi_{2} \partial \pi_{1}} & \frac{\partial^{2} S_{\text {eff }}}{\partial \pi_{2}^{2}} & \frac{\partial^{2} S_{\text {eff }}}{\partial \pi_{2} \partial \pi_{3}} \\
\frac{\partial^{2} S_{\text {eff }}}{\partial \pi_{3} \partial \sigma} & \frac{\partial^{2} S_{\text {eff }}}{\partial \pi_{3} \partial \pi_{1}} & \frac{\partial^{2} S_{\text {eff }}}{\partial \pi_{3} \partial \pi_{2}} & \frac{\partial^{2} S_{\text {eff }}}{\partial \pi_{3}^{2}}
\end{array}\right),
$$

and also evaluated at the saddle point. This matrix is a measure of the curvature of the potential at the saddle point, i.e. the magnitude of the fluctuations of the fields around this point, and it is thus related to the masses of the effective excitations.

Using the NJL model partition function, we can now write Eq. (2) as

$$
\left\langle e^{2 i \theta}\right\rangle=F\left(\mu_{q}, T\right) e^{-\beta V \Delta \Omega\left(\mu_{q}, T\right)}
$$

where the prefactor $F$ is the square root of the ratio between the Hessian determinants in the $1+1^{*}$ and the $1+1$ theories, i.e.

$$
F\left(\mu_{q}, T\right) \equiv \sqrt{\frac{\operatorname{det} H\left(\mu=0, \delta \mu=\mu_{q}\right)}{\operatorname{det} H\left(\mu=\mu_{q}, \delta \mu=0\right)}},
$$

while $\Delta \Omega$ is the difference in thermodynamic potential between the two cases:

$$
\Delta \Omega\left(\mu_{q}, T\right) \equiv \Omega\left(\mu=\mu_{q}, \delta \mu=0\right)-\Omega\left(\mu=0, \delta \mu=\mu_{q}\right) .
$$

Here, $\mu_{q}$ is used to denote the quark chemical potential, so that $\mu_{q}=\mu_{B} / 3$.

In the numerical analysis, all momentum integrals have been regulated using a sharp ultraviolet cutoff of $\Lambda=$ $651 \mathrm{MeV}$. We have taken the bare quark mass and the coupling constant to be $m_{0}=5.5 \mathrm{MeV}$ and $G=2.12 / \Lambda^{2}$, respectively, and set the number of colors to $N_{c}=3$. With this parameter set, the model reproduces the pion mass $m_{\pi}=139 \mathrm{MeV}$, the pion decay constant $f_{\pi}=94$ $\mathrm{MeV}$, and a chiral condensate of $\langle\bar{u} u\rangle=-(250 \mathrm{MeV})^{3}$ in the vacuum.

In the plots of the average phase factor $\left\langle e^{2 i \theta}\right\rangle$ we have set the three-volume $V$ equal to $L^{3}$, where $L=4 / T$. Thus, the full four-volume is

$$
\beta V=\frac{64}{T^{4}}
$$

This choice is inspired by lattice QCD, and corresponds to a lattice size of $4 \times 16^{3}$.

\section{THE IMPORTANCE OF THE PREFACTOR}

In Fig. 1] we show the chiral condensate as a function of function of $T / m_{\pi}$ and $\mu / m_{\pi}$. In Fig. 2 we show the chiral condensate as a function of $T / m_{\pi}$ and $\mu / m_{\pi}$. Comparing the two, we immediately see that the free energies must differ for $\mu, \delta \mu>m_{\pi} / 2$ and low $T$. Indeed this is confirmed by computing directly the difference of the two free energies. This is shown in Fig. 3. This difference is due to the formation of a pion condensate, $\rho$ in the phase-quenched theory. In Fig. 4 we show a plot of the pion condensate as a function of $T / m_{\pi}$ and $\delta \mu / m_{\pi}$. As expected, the pion condensation sets in at $\delta \mu=m_{\pi} / 2$ for $T=0$. As the temperature is increased it takes a somewhat larger chemical potential to trigger the formation of the pion condensate. As the pion condensate forms, the chiral condensate starts dropping to zero.

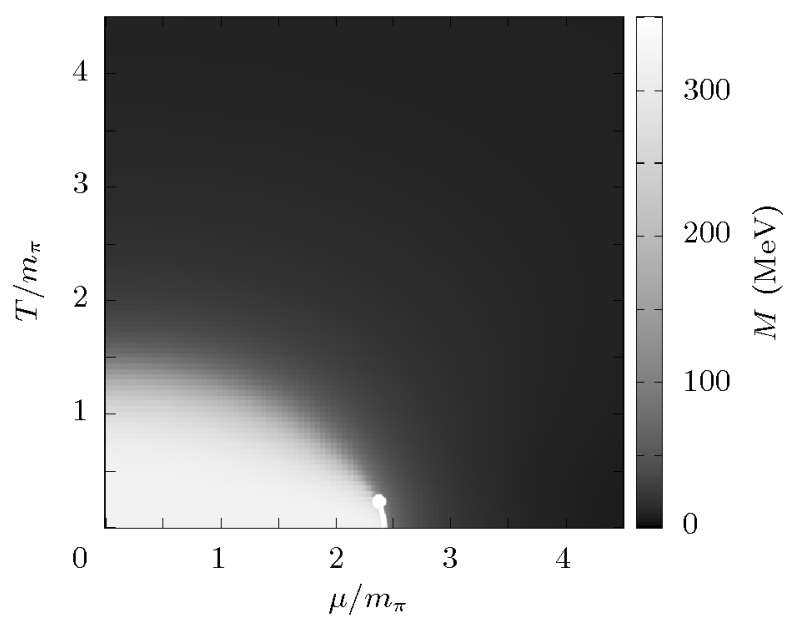

Figure 1: The chiral condensate $M$ in the $1+1$ theory with $\alpha=0$ as a function of $T / m_{\pi}$ and $\mu / m_{\pi}$. The curve marks the first order phase transition, while the dot marks the critical point.

We conclude from this that the average phase factor is exponentially small when there is a pion condensate in the phase-quenched theory, cf. Eq. (30). However, when the pion condensate vanishes in the phase-quenched theory, the mean field NJL free energy is equal to that for the ordinary theory, i.e. $\Delta \Omega\left(\mu_{q}, T\right)=0$ if $\rho=0$. The average phase factor, Eq. (30), is then dominated by the prefactor $F$ throughout an extended region of the $\left(\mu_{q}, T\right)$ plane.

The Hessian matrix measures the curvature of the potential at the minimum and can hence be interpreted as a product of quasiparticle masses. For low temperatures where the pions dominate the partition function, the prefactor is simply [27]

$$
F=1-\left(\frac{2 \mu_{q}}{m_{\pi}}\right)^{2} .
$$

This low-temperature behavior of the prefactor is con- 


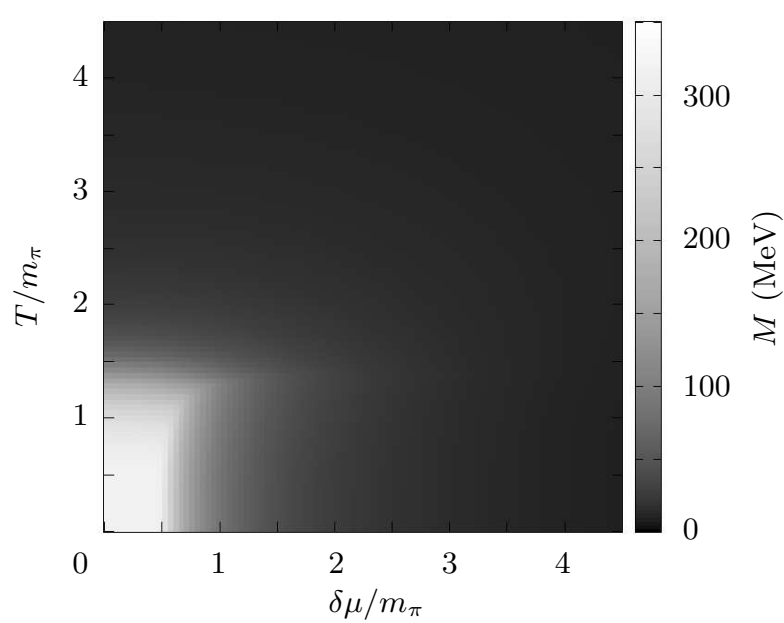

Figure 2: The chiral condensate $M$ in the $1+1^{*}$ as a function of $T / m_{\pi}$ and $\delta \mu / m_{\pi}$ theory with $\alpha=0$.

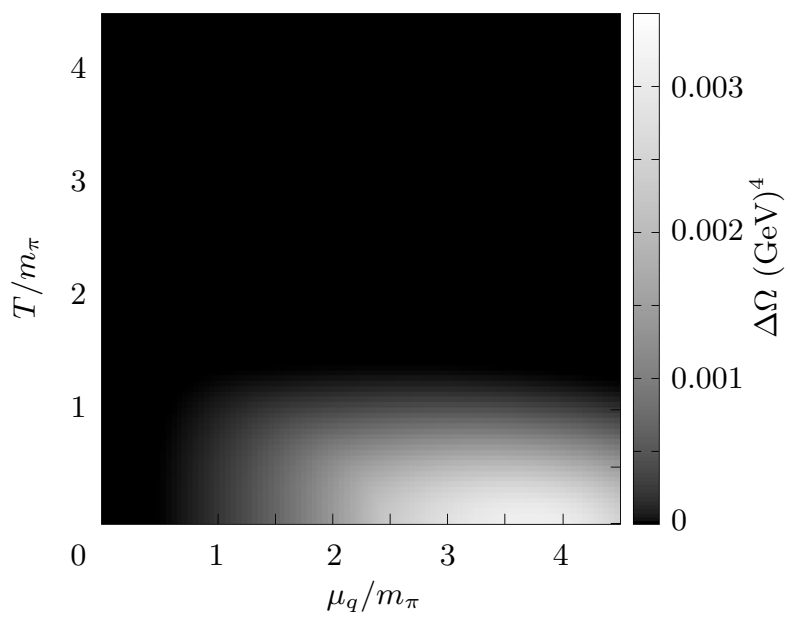

Figure 3: The difference in the free energy densities $(\Delta \Omega)$, between the $1+1$ theory and the $1+1^{*}$ theory as a function of $T / m_{\pi}$ and $\mu / m_{\pi}$ with $\alpha=0$.

firmed by the NJL model computation: In Fig. 5 we show the prefactor $F$ as predicted in chiral perturbation theory (solid curve) and the prediction by the NJL model (crosses).

The full plot of the average phase factor in the $\left(\mu_{q}, T\right)$ plane is given in Fig. 6. We see that indeed the sign problem is exponentially bad in a region corresponding to the region of pion condensation in the phase quenched theory. Before reaching this the prefactor dominates and the average phase factor drops smoothly from one to zero.

The full dominance of the prefactor outside the pioncondensed region is an artifact of the mean-field approximation. This has been demonstrated for low temperatures in chiral perturbation theory, where one-loop pion effects give rise to a nonzero value of $\Delta \Omega$ also where the

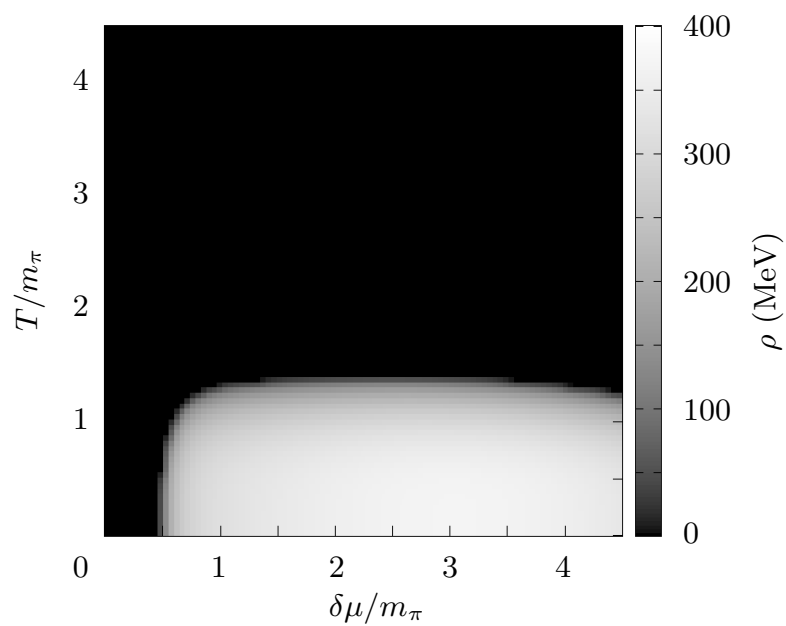

Figure 4: The pion condensate $\rho$ in the $1+1^{*}$ theory as a function of $T / m_{\pi}$ and $\delta \mu / m_{\pi}$. with $\alpha=0$.

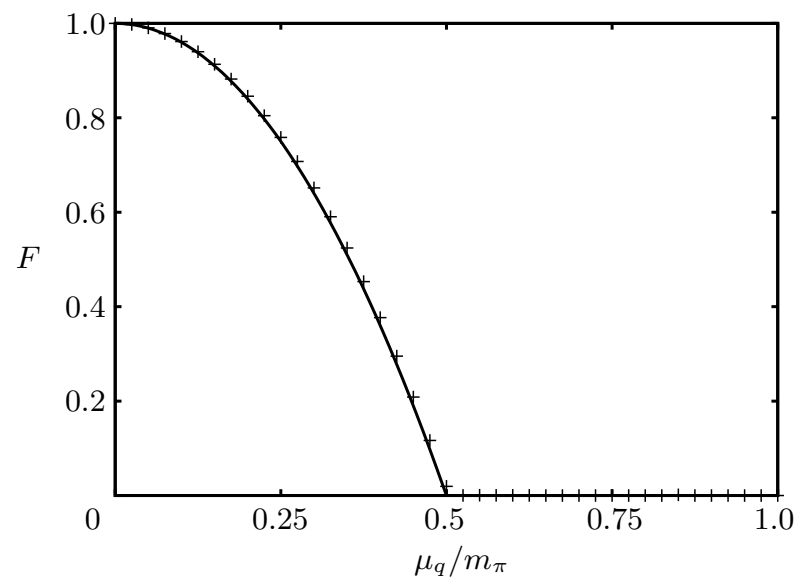

Figure 5: The prefactor $F$ for $T=1 \mathrm{MeV}$ and $\alpha=0$ (crosses), and the prediction $1-\left(2 \mu_{q} / m_{\pi}\right)^{2}$ from Ref. [27] (line). Note that the choice of $T=1 \mathrm{MeV}$ instead of $T=0$ is purely for computational convenience.

pion condensate vanishes [27]. By comparison to lattice QCD measurements of the phase factor, it is seen that both the prefactor and the one-loop contribution to $\Delta \Omega$ are relevant for the standard lattice volumes used [28]. Nevertheless, the overall trend for the average phase factor also above the critical temperature is in agreement with lattice studies [48].

\section{THE EFFECT OF $U_{A}(1)$ SYMMETRY BREAKING}

Instanton effects are responsible for the $U_{A}(1)$ axial anomaly in QCD and is known [43] to have a strong influence on order of the phase transition at zero chemical potential. In Ref. [44] it was demonstrated that the 

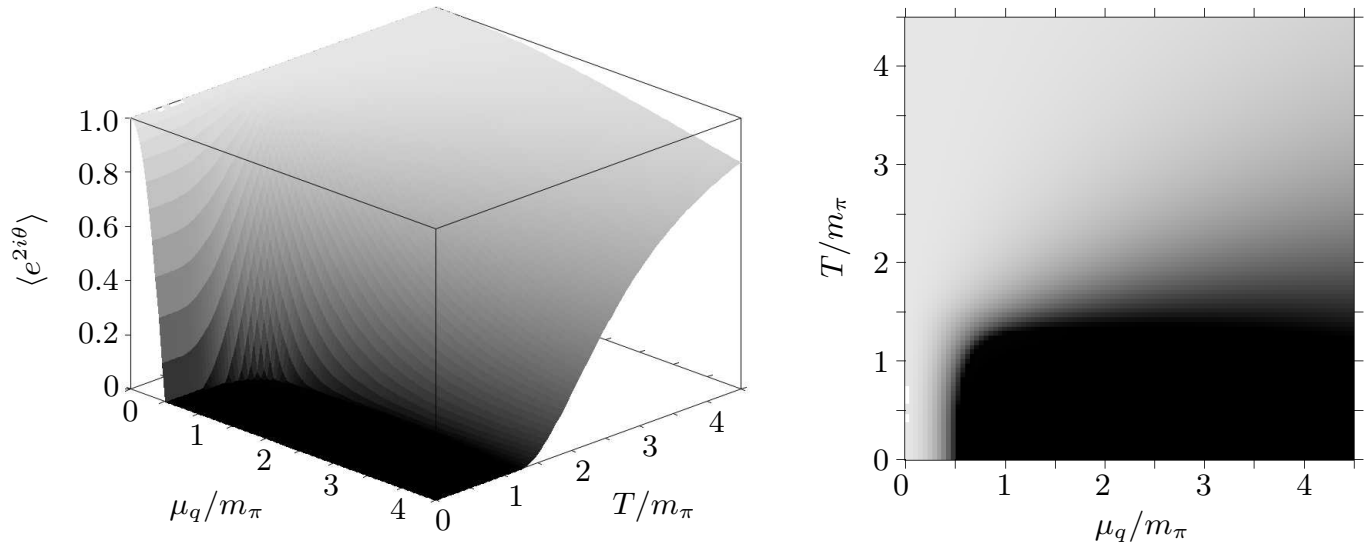

Figure 6: The average phase factor $\left\langle e^{2 i \theta}\right\rangle$ as a function of $\mu_{q} m_{\pi}$ and $T / m_{\pi}$ for $\alpha=0$.

strength of the $U_{A}(1)$ axial anomaly is also important for nonzero $\mu$ in the three-flavor NJL model. Here we consider the effects of the anomaly on the phase factor within the two-flavor NJL model. As in Ref. [44] we work with a $\mu_{q}$-dependent $\alpha$,

$$
\alpha=\frac{1}{2} e^{-\mu_{q}^{2} / \mu_{0}^{2}},
$$

so that the $U_{A}(1)$ symmetry is restored at $\mu_{q}^{2} \gg \mu_{0}^{2}$. The scale $\mu_{0}$ introduces a new parameter in the NJL model. The form (35) is motivated by the Gaussian suppression of the instanton density due to Debye screening at large values of the chemical potential.

The effects of $\alpha$ on the phase diagram of the two-flavor NJL model at nonzero $\mu$ and $\delta \mu$ have been discussed in Ref. [39]. There, the focus was to demonstrate that the splitting of the $\langle\bar{u} u\rangle$ and $\langle\bar{d} d\rangle$ transitions induced by $\delta \mu$ is wiped out by the breaking of $U_{A}(1)$ symmetry. Here the setup is somewhat different since we do not have $\mu$ and $\delta \mu$ nonzero at the same time; recall that we are looking at the ratio $Z_{1+1} / Z_{1+1^{*}}$. This implies that $\langle\bar{u} u\rangle=\langle\bar{d} d\rangle$. Thus there is no effects of $\alpha$ in the free energies of the mean-field two-flavor NJL model considered here, and the dependence of the average phase factor on $\alpha$ is entirely due to the $\alpha$-dependence of the prefactor $F$.

In Fig. 7 we have plotted the average phase factor for four different temperatures, both with and without axial symmetry breaking. For the plot we have chosen $\mu_{0}=m_{\pi}$. We observe that there is no effect of $\alpha$ for low temperature whereas the $U_{A}(1)$ breaking increases the average phase factor slightly for higher temperatures.

From a Ginzburg-Landau perspective, it is easy to see that the effect of $U_{A}(1)$ breaking on the phase diagram in the two-flavor theory is small, since the $U_{A}(1)$ breaking only gives a quadratic term in the order parameter. This term is already present at $\alpha=1 / 2$. For $N_{f}=3$ the $U_{A}(1)$ breaking induces a cubic term in the GinzburgLandau free energy - see for example Ref. 44]. This term potentially has a much larger effect on the position of the

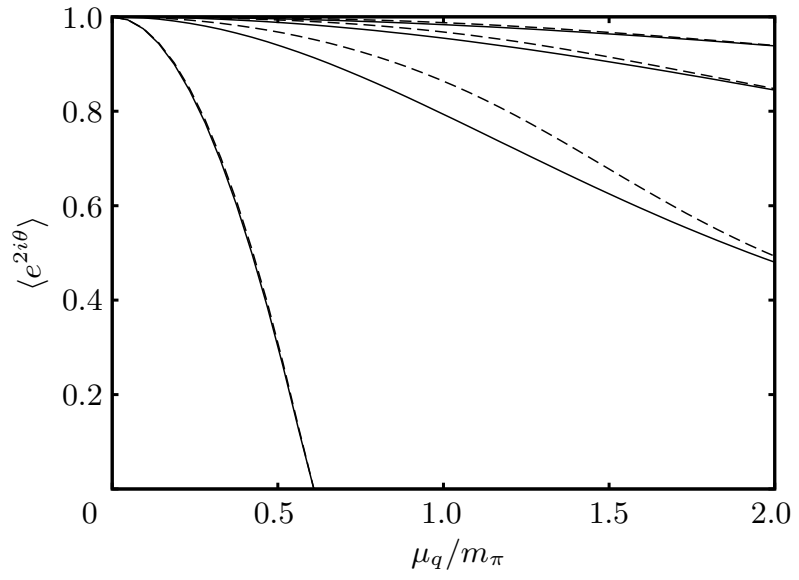

Figure 7: The average phase factor $\left\langle e^{2 i \theta}\right\rangle$ for $T=m_{\pi}$ (lower), $T=2 m_{\pi}, T=3 m_{\pi}$, and $T=4 m_{\pi}$ (upper), when $\alpha=0$ (solid curves) and $\alpha=\frac{1}{2} e^{-\mu_{q}^{2} / m_{\pi}^{2}}$ (dashed curves).

endpoint. Indeed this is what was observed for the threeflavor NJL model in Ref. [44].

\section{THE ENDPOINT AND THE SIGN PROBLEM}

The endpoint of the first order chiral phase transition has been studied intensively in recent years. One conclusion which has emerged is that the location of the endpoint in the $\left(\mu_{q}, T\right)$ plane is extremely sensitive to the value of the quark masses and the fate of the $U_{A}(1)$ breaking. In order for lattice QCD to be able to reach the location of the endpoint in the $\left(\mu_{q}, T\right)$ plane, before the numerical noise due to the sign problem wipes out the signal, the endpoint must be located outside the region where the average phase factor is exponentially suppressed. Whether or not this is the case is un- 

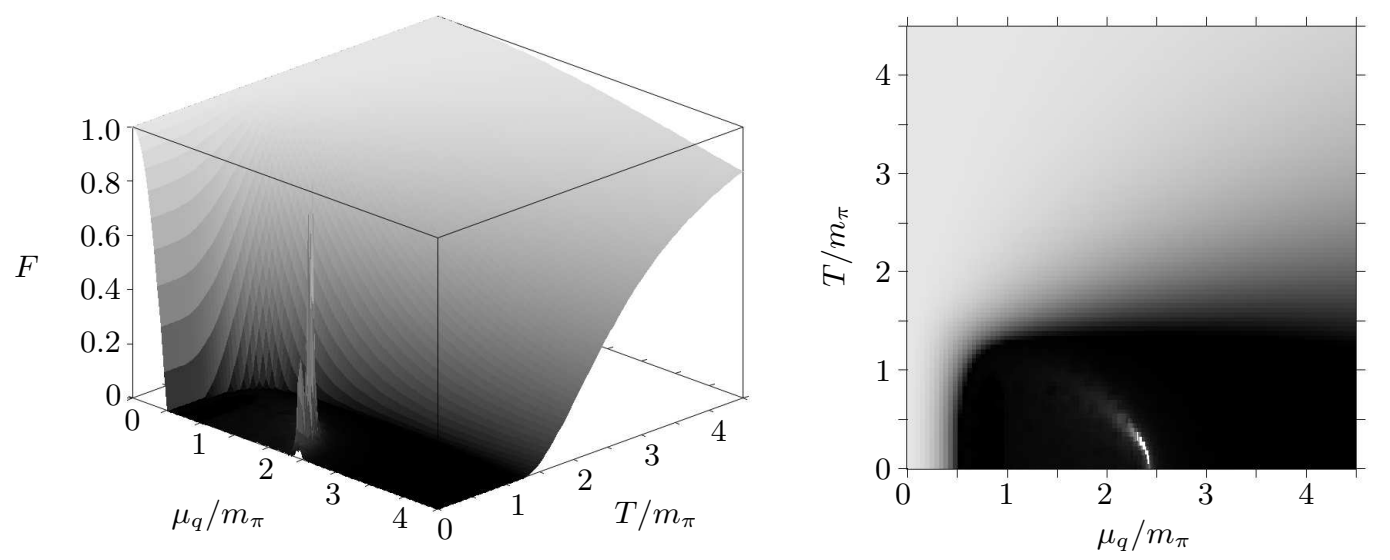

Figure 8: The prefactor $F$ as a function of $\mu_{q} / m_{\pi}$ and $T / m_{\pi}$ for $\alpha=0$. Note the spike at the critical point, which extends far above the maximum value of $F$.

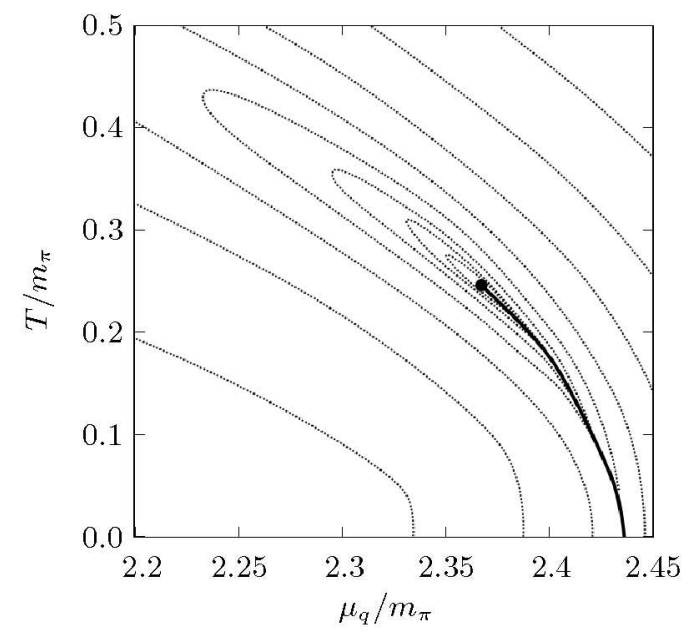

Figure 9: Contour plot of the prefactor $F$ around the critical endpoint, with $\alpha=0$. The solid line is the first-order phase transition, and the dot marks the endpoint. The dotted lines are contours of equal $F$, each separated by a power of $e$.

clear. Both the position of the endpoint as well as the region where the average phase factor is exponentially suppressed depend on the quark masses. In chiral Random Matrix Models 40, 45] and in the two-flavor NJL model, the location of the endpoint is always inside the exponentially suppressed region. The endpoints found by multi-parameter reweighting techniques in both Refs. 19. and 20] are located where the exponential suppression sets in [46]. Here we discuss how the sign problem is correlated with the critical endpoint.

If the endpoint is located in the region where the average phase factor is exponentially suppressed it is not accible by current lattice methods. If, however, the endpoint is located at small values of $\mu_{q}$, i.e. $\mu_{\text {endpoint }}<m_{\pi} / 2$, then the story is somewhat different. Two scenarios are possible depending on what happens in the phase- quenched theory:

1) The phase-quenched theory has an endpoint at exactly the same location as the full theory. In this case the average phase factor will not show any particular sign of the endpoint.

2) The endpoint in the phase-quenched theory is not at the same location as in the full theory. In this case the average phase factor can grow as one approaches the critical endpoint. The reason is simple: just like the massless mode in the pion-condensed theory drives the prefactor $F$ to zero, see. Fig. 5 , the massless $\sigma$ at the endpoint will induce a pole in the prefactor since the Hessian of the $1+1$ theory is in the denominator, cf. Eq. (3). We can illustrate this by looking at the prefactor, $F$, in the two-flavor NJL model. In Fig. 8, we have shown the prefactor $F$ as a function of $\mu_{q} / m_{\pi}$ and $T / m_{\pi}$. We see that at the endpoint in the $1+1$ theory the massless $\sigma$ has produced a peak in the exponential prefactor. In this case the peak is suppressed by the exponential factor even with a small volume. If, however, the endpoint is located outside the exponentially suppressed region, the peak due to the massless $\sigma$ may survive to much larger values of $V$. This is because the free energy difference $\Delta \Omega$ can be much smaller here, since a cusp in $\Omega_{1+1}$ does not necessarily induce a large $\Delta \Omega$. Of course, it must always be true that $0 \leq\left\langle e^{2 i \theta}\right\rangle \leq 1$, so the pole and the exponential factor must always be considered together to ensure this.

As mentioned above it is unfortunately not possible to fully illustrate this most interesting scenario in the twoflavor NJL model used here. However, it is quite possible that it could be realized in the three-flavor NJL model with $U_{A}(1)$ axial symmetry breaking.

One of the characteristic features of the critical point is the divergence of the correlation length $\xi$. Since the correlation length is inversely proportional to the sigma mass, while the Hessian matrix is roughly proportional to the squares of the masses, we expect that as we approach 
the endpoint,

$$
F \sim \xi
$$

In Fig. 9 we have shown a contour plot of the prefactor $F$ in the region immediately surrounding the endpoint. A comparison of this figure to Fig. 3 of Ref. [47], which is a similar plot of the correlation length $\xi$, seems to confirm Eq. (36). A notable feature of this plot is the fact that the prefactor - specifically, the contribution from the prefactor to the $Z_{1+1}$ partition function-is discontinuous across the phase transition. This seems to indicate that there is a zeroth-order phase transition in the theory. Fortunately, this discontinuity is entirely due to corrections to the partition function of order $1 / V$, so there is no inconsistency in the infinite-volume limit.

\section{CONCLUSIONS}

We have presented the first study of the average phase factor of the complex QCD fermion determinant within a two-flavor NJL model. This study extends previous studies in several directions: We have considered temperatures not accessible to chiral perturbation theory, we have considered the effects of the $U_{A}(1)$ anomaly on the sign problem and we have discussed the interplay between the sign problem and the critical endpoint of the chiral phase transition. The NJL model certainly does not capture all effects of QCD and one should not take the precise numerical values given here as firm predictions. However, several general conclusions can be drawn from this study of the NJL model.

First, we have seen that the exponential prefactor in the mean-field approximation dominates for a large range of $\mu_{q}$ when the temperature is above the critical one. This is in complete agreement with the result obtained from a mean field random matrix approach [40]. For lattice QCD, this means that one should expect that the masses of the effective excitations have a large effect on the strength of the sign problem.

Second, the average phase factor depends on the $U_{A}(1)$ axial anomaly when the temperature is close to or above the critical one. The present study indicates that the sign problem may become milder when $U_{A}(1)$ symmetry breaking is taken into account.

Third, we have discussed how the massless mode at the endpoint can drive the average phase factor to larger values. Since this indicates a milder sign problem at the endpoint, this observation is highly relevant for lattice investigations of the endpoint. Of course the free energies in the phase-quenched and the full theory must be slightly different if the latter is to have an endpoint while the former does not. However, this difference need not be numerically large (as it is in the pion condensed region) and the exponential suppression of the prefactor thus may only set in at large values of $V$. Unfortunately is it not possible to study this effect fully within the twoflavor NJL model.

The order of the QCD phase transition depends on the number of flavors as well their masses [49]. It would be therefore very interesting to extend this study to the three-flavor case where it also known that the effects of the $U_{A}(1)$ axial anomaly are greater.

\section{Acknowledgments}

J. O. A. and L. T. K. would like to thank the Niels Bohr International Academy, where this work was initiated, for kind hospitality. K. S. would like to thank Thomas Schäfer for discussions. This work was supported by the Danish Natural Science Research Council (K. S.).
[1] I. R. Klebanov, Nucl. Phys. B 262, 133 (1985).

[2] A. D. Jackson and J. J. M. Verbaarschot, Nucl. Phys. A 484, 419 (1988).

[3] R. Rapp, T. Schafer, E. V. Shuryak and M. Velkovsky, Phys. Rev. Lett. 81, 53 (1998) arXiv:hep-ph/9711396; M. G. Alford, K. Rajagopal and F. Wilczek, Phys. Lett. B 422, 247 (1998) arXiv:hep-ph/9711395.

[4] L. McLerran and R. D. Pisarski, Nucl. Phys. A 796, 83 (2007) arXiv:0706.2191 [hep-ph]].

[5] K. Rajagopal and F. Wilczek, arXiv:hep-ph/0011333

[6] J. B. Kogut and D. K. Sinclair, Phys. Rev. D 70, 094501 (2004) arXiv:hep-lat/0407027.

[7] J. B. Kogut and D. K. Sinclair, Phys. Rev. D 77, 114503 (2008) arXiv:0712.2625 [hep-lat]].

[8] P. de Forcrand, M. A. Stephanov and U. Wenger, PoS LAT2007, 237 (2007) arXiv:0711.0023 [hep-lat]].

[9] M. G. Alford, A. Kapustin and F. Wilczek, Phys. Rev. D 59, 054502 (1999) arXiv:hep-lat/9807039.

[10] P. de Forcrand and O. Philipsen, PoS LATTICE2008,
208 (2008) arXiv:0811.3858 [hep-lat]]; JHEP 0811, 012 (2008) arXiv:0808.1096 [hep-lat]].

[11] P. de Forcrand and O. Philipsen, Nucl. Phys. B 642, 290 (2002) arXiv:hep-lat/0205016.

[12] M. D'Elia and M. P. Lombardo, Phys. Rev. D 67, 014505 (2003) arXiv:hep-lat/0209146.

[13] P. de Forcrand and O. Philipsen, Nucl. Phys. B 673, 170 (2003) arXiv:hep-lat/0307020.

[14] R. V. Gavai and S. Gupta, Phys. Rev. D 68, 034506 (2003) arXiv:hep-lat/0303013.

[15] C. R. Allton et al., Phys. Rev. D 66, 074507 (2002) arXiv:hep-lat/0204010].

[16] C. R. Allton, S. Ejiri, S. J. Hands, O. Kaczmarek, F. Karsch, E. Laermann and C. Schmidt, Phys. Rev. D 68, 014507 (2003) arXiv:hep-lat/0305007.

[17] C. R. Allton et al., Phys. Rev. D 71, 054508 (2005) arXiv:hep-lat/0501030.

[18] I. M. Barbour, S. E. Morrison, E. G. Klepfish, J. B. Kogut and M. P. Lombardo, Nucl. Phys. Proc. 
Suppl. 60 A (1998) 220.

[19] Z. Fodor and S. D. Katz, JHEP 0203, 014 (2002) arXiv:hep-lat/0106002.

[20] Z. Fodor and S. D. Katz, JHEP 0404, 050 (2004) arXiv:hep-lat/0402006.

[21] F. Karsch and H. W. Wyld, Phys. Rev. Lett. 55, 2242 (1985).

[22] J. Flower, S. W. Otto and S. Callahan, Phys. Rev. D 34, 598 (1986).

[23] J. Ambjorn, M. Flensburg and C. Peterson, Nucl. Phys. B 275, 375 (1986).

[24] G. Aarts and I. O. Stamatescu, JHEP 0809, 018 (2008) arXiv:0807.1597 [hep-lat]]; G. Aarts, arXiv:0810.2089 [hep-lat]; arXiv:0902.4686] [hep-lat].

[25] Z. Fodor, S. D. Katz and C. Schmidt, JHEP 0703, 121 (2007) arXiv:hep-lat/0701022.

[26] S. Ejiri, Phys. Rev. D 77, 014508 (2008) arXiv:0706.3549 [hep-lat]].

[27] K. Splittorff and J. J. M. Verbaarschot, Phys. Rev. D 75, 116003 (2007) arXiv:hep-lat/0702011.

[28] K. Splittorff and J. J. M. Verbaarschot, Phys. Rev. D 77, 014514 (2008) arXiv:0709.2218 [hep-lat]].

[29] J. C. R. Bloch and T. Wettig, arXiv:0812.0324 [hep-lat].

[30] K. Splittorff and B. Svetitsky, Phys. Rev. D 75, 114504 (2007) arXiv:hep-lat/0703004.

[31] M. D'Elia and F. Sanfilippo, Phys. Rev. D 80, 014502 (2009) arXiv:0904.1400 [hep-lat]].

[32] A. Barducci, R. Casalbuoni, G. Pettini and L. Ravagli, Phys. Rev. D 69, 096004 (2004) arXiv:hep-ph/0402104.

[33] C. Ratti and W. Weise, Phys. Rev. D 70, 054013 (2004) arXiv:hep-ph/0406159.

[34] L. y. He, M. Jin and P. f. Zhuang, Phys. Rev. D 71,
116001 (2005) arXiv:hep-ph/0503272.

[35] D. Ebert and K. G. Klimenko, J. Phys. G 32, 599 (2006) arXiv:hep-ph/0507007.

[36] S. Lawley, W. Bentz and A. W. Thomas, Phys. Lett. B 632, 495 (2006) arXiv:nucl-th/0504020.

[37] J. O. Andersen and L. Kyllingstad, arXiv:hep-ph/0701033

[38] H. Abuki, R. Anglani, R. Gatto, M. Pellicoro and M. Ruggieri, Phys. Rev. D 79, 034032 (2009) arXiv:0809.2658 [hep-ph]].

[39] M. Buballa, Phys. Rept. 407, $205 \quad$ (2005) arXiv:hep-ph/0402234.

[40] J. Han and M. A. Stephanov, Phys. Rev. D 78, 054507 (2008) arXiv:0805.1939 [hep-lat]].

[41] K. Splittorff and J. J. M. Verbaarschot, Nucl. Phys. B 683, 467 (2004) arXiv:hep-th/0310271.

[42] J. M. Moller, arXiv:0908.1642 [hep-lat].

[43] R. D. Pisarski and F. Wilczek, Phys. Rev. D 29, 338 (1984).

[44] J. W. Chen, K. Fukushima, H. Kohyama, K. Ohnishi and U. Raha, arXiv:0901.2407 [hep-ph].

[45] B. Klein, D. Toublan and J. J. M. Verbaarschot, Phys. Rev. D 68, 014009 (2003) arXiv:hep-ph/0301143.

[46] K. Splittorff, PoS LAT2006, 023 (2006) arXiv:hep-lat/0610072 ; arXiv:hep-lat/0505001

[47] M. A. Stephanov, Phys. Rev. Lett. 102, 032301 (2009) arXiv:0809.3450 [hep-ph]].

[48] J. Danzer, C. Gattringer, L. Liptak and M. Marinkovic, arXiv:0907.3084 [hep-lat].

[49] M. A. Stephanov, PoS LAT2006, 024 (2006). arXiv:hep-lat/0701002. 